\title{
VIRTUAL TERRESTRIAL LASER SCANNER SIMULATOR FOR DIGITALISATION OF TEACHING ENVIRONMENT: CONCEPT AND FIRST RESULTS
}

\author{
Maria Chizhova ${ }^{1}$, Darius Popovas ${ }^{2}$, Denys Gorkovchuk ${ }^{3}$, Julia Gorkovchuk ${ }^{4}$, Mona Hess ${ }^{5}$, Thomas Luhmann ${ }^{6}$ \\ ${ }^{1}$ University of Bamberg, Institute of Archaeology, Heritage Sciences and Art History, Germany - maria.chizhova@uni-bamberg.de \\ 2 Jade University of Applied Sciences, Institute for Applied Photogrammetry and Geoinformatics, Oldenburg, Germany - \\ darius.popovas@jade-hs.de \\ ${ }^{3}$ Kiev National University for Construction and Architecture, SPM3D LLC, Kyiv, Ukraine - denys.gorkovchuk@spm3d.com \\ ${ }^{4} \mathrm{Kiev}$ National University for Construction and Architecture, Kyiv, Ukraine - gorkovchukjulia@gmail.com \\ ${ }^{5}$ University of Bamberg, Institute of Archaeology, Heritage Sciences and Art History, Germany - mona.hess@uni-bamberg.de \\ ${ }^{6}$ Jade University of Applied Sciences, Institute for Applied Photogrammetry and Geoinformatics, Oldenburg, Germany - \\ luhmann@jade-hs.de
}

Commission V, WG V/7

KEY WORDS: Simulator, laser scanning, point cloud, virtual scanner, game engine, curriculum development, online training.

\begin{abstract}
We are presenting a concept and first results of a Terrestrial Laser Scanner simulator - a tool, which could be a valuable educational tool for geomatics and engineering students. The main goal of the VirScan3D project is to cover engineering digitisation by two major project parts: 1) Development of a virtual environment for creation and processing of digital 3D scan data; 2) Digital teaching and e-learning material with interactive tools and practical experiences. Part 1 will be solved through the development of a virtual system that allows users to create realistic data in the absence of a real measuring device. At the time of writing (April 2020) all higher education teaching and learning worldwide has been changed to online delivery due to the Covid pandemic. However, this has affected the courses that involve working with equipment and - consequently - require the presence of students and devices. In this project, the development of such equipment simulator proposes a real and feasible solution, which allows undisturbed continuing the teaching.
\end{abstract}

The virtual scanner will be based on existing software packages. It will be part of a general e-learning concept that will be implemented in partner universities. Part 2 is addressed through an integrated teaching concept that includes interactive learning tools (for both teachers and students) and practical exercises (for example project weeks, summer schools) where the theoretical and virtual education is connected to practical experience with real instrumentation and data. One outcome of the experimental work is additional e-learning material based on the experiences and results of the project work.

The prototype implementation of the virtual laser scanner is realized within a game engine, which allows for fast and easy 3D visualisation and navigation. Within this environment, the user can freely navigate and define suitable scanning positions/stations. At each scanning station a simulated scan is performed, which is based on the technical specifications of a real scanner. The mathematical solution is based on 3D line intersection with the virtual 3D surface including noise and colour as well as an intensity simulation. As a result, 3D point clouds for each station are generated, which will be further processed for registration and modelling using standard software packages.

\section{INTRODUCTION}

\subsection{Project background}

In the areas of environmental planning, building construction, infrastructure maintenance or cultural heritage conservation, the use of digital geospatial information has significantly grown during the last decade, for instance in the field of building information modelling (BIM). Location-based data and geo-information in general are nowadays also a key factor in economy such as logistics, energy, communication or internet business. Specifically, new instruments for digital recording and processing tools are increasingly established in those application areas.
Since digitalisation is one of the major world-wide trends, the teaching curricula, related to digitalisation in engineering practice (e.g. geoinformation sciences, cadastral mapping, geodesy) should educate their students according to current needs and demands from the professional market and industry. State-of-the art technologies in these fields (e.g. 3D laser scanning, photogrammetry, UAVs) lead to large amounts of digital data and are difficult to process, analyse and transform into useful information. For this purpose, appropriate tools and workflows have to be provided, otherwise neither teachers nor students have realistic chances to adapt to new methods in due time, and they will not be able to contribute to further developments.

However, this objective is hard to achieve, since there is a lack of knowledge among many teachers, out-of-date equipment 
(hardware and software), insufficient ICT infrastructure and insufficient finances for necessary investments. Furthermore, development of recording instruments has a rapid change cycle of ca. five years. For instance, expensive terrestrial laser scanners are very rare in higher education institutions. In addition, a strong interdisciplinary approach should be followed since these technologies are relevant in many other subjects such as civil engineering, machine construction, cultural heritage or informatics (Hess et al., 2017).

Since the use of such devices and the subsequent data processing are not trivial, we propose that students can be trained on virtual systems that are very cost-effective, flexible and future oriented.

In the current situation, with COVID-19 pandemic ongoing, all teaching and learning efforts have been switched to online delivery worldwide. However, this has affected the courses that involve working with equipment and on-site and - consequently - require the presence of students. In this case, the development of our equipment simulator is a real solution, which allows undisturbed continuation of teaching.

\subsection{Project idea}

We are presenting a concept and first results of a Terrestrial Laser Scanner simulator - a tool, which could be a valuable educational tool for geomatics and engineering students.

This project with the acronym VirScan3D is funded from 2019 to 2021 by DAAD (German Academic Exchange Service) within a program for "Supporting the internationalisation of Ukrainian universities: German-Ukrainian higher education institution collaborations". The project group consists of two German universities and one Ukrainian university based on a long-term cooperation that has started in 2002. As one part of the ongoing cooperation, joint student exchange projects have been established (Kravchenko et al., 2016). Certainly, due to its high relevance in online-teaching and learning this project will continue on beyond the funded run-time.

\subsubsection{Educational and technological aspects of the current situation in Higher Education in Ukraine}

Nowadays growing focus on education and its quality is the key trend in Ukraine and worldwide. The International Organization for Standardization (ISO) defines the quality of education as a set of properties and characteristics of the educational process, which provide the ability to meet the educational needs of educational services consumers. It emphasizes the importance of assessing the quality achieved by all consumers of educational services - both direct (student, student) and indirect (employer, state, community, society) according to ISO 9000:2005.

According to ISO 9000:2005, the main factors that ensure the quality of technical education are:

highly professional training of teaching subjects, educational and methodological support of the preparation process and its compliance with modern requirements,

- the use of modern (digital) educational technologies (active teaching methods, internet technologies, etc.) in the educational process, and

- $\quad$ suitable logistical support for the preparation process.
The qualitative training of engineers in the field of geodesy, geoinformatics and photogrammetry is based on implementation of state-of-the-art equipment and digital technologies into the educational process. Leading manufacturers of hardware and software (Leica, Topcon, Trimble, Agisoft, ESRI, etc.) offer loyalty programs and special sales conditions for educational and scientific institutions to solve this problem. However, in developing countries it is hard to achieve, since there is a lack of knowledge among many teachers, out-of-date equipment (hardware and software), insufficient ICT infrastructure and insufficient finances for necessary investments. The use of expensive equipment, such as mobile mapping systems, is not economically feasible for training purposes. But only the opportunity to acquire practical skills with modern tools for professionals of all levels will lead to widespread implementation of technology in the production process. Among the advanced methods of digitalisation of professional education, techniques of virtual and augmented reality are very effective. This is confirmed by research on the high efficacy of such training approaches (Allcoat, Mühlenen, 2018) and the rapid development of the VR and AR training market in the world according to the $\mathrm{ABI}$ research in augmented and virtual reality in education (ABI 2019).

\subsubsection{Impact from development and realisation of the VirScan3D simulator for Ukrainian universities}

The application of a modern geodetic equipment using virtual systems can be very cost-effective, flexible and future oriented. Development of a virtual system will allow users to create data in the absence of a real measuring device. Terrestrial laser scanners remain inaccessible in Ukraine (typically used in specialized commercial organizations). However, the use of such devices and further processing of data is already an important skill in the professional activity of a wide range of specialists. The results of 3D scanners - point clouds - are used for various purposes, in particular for the creation of 3D models of buildings and structures, CAD modelling for parts production, metrology / quality control and for use in numerous applications of visualization, animation, rendering and large individualized production. Accordingly, the target customers of the virtual environment for creating and processing digital 3Dscanning data is a wide range of users of the following technologies: architects, designers, engineers, builders, surveyors, land surveyors, GIS specialists. Students will be able to learn skills by means of virtual systems that are cost-effective (free in open environments), flexible, and future-oriented. The virtual scanner simulates commercially available tools with realistic user interfaces and creates $3 \mathrm{D}$ point clouds according to individual specifications and settings. Any digital 3D model (such as a cultural or construction object) that is virtually scanned in a digital environment can be used as training object. With this system, teachers and students will be able to learn how to manage such systems, generate realistic big data and complete the entire data processing chain.

A virtual learning environment will solve the problem of providing high-precision (and high-value) devices in accordance with professional standards. It is relevant for the specialists in the fields of geodesy, photogrammetry, GIS and land management as well as neighborhood disciplines (architecture and cultural heritage digitalization, computer vision, mathematics etc.) 


\subsubsection{Worldwide impact from equipment simulation}

The situation mentioned in previous sections, i.e. lack of financial support, lack of knowledge and ICT recourses, is quite common in many developing countries. Even in well-developed countries, it is hard to keep pace with rapid technological progress, so the VirScan3D simulator can be efficiently utilized in teaching processes of HEI (higher education institutions). Furthermore, the laser scanner simulator can be used in lifelong learning scenarios (e.g. for professionals with ongoing employment), where new trends require knowledge of 3D spatial data collection and processing. Nowadays, such fields as architecture, BIM, archeology, cultural heritage, construction, etc., more widely using 3D scanning technology, therefore additional training of specialists is essential and virtual laser scanner simulator can successfully be used.

In addition, a virtual scanning system may be very effective in times like the recent COVID-19 crisis, where access to real equipment may be restricted.

During the project period (2019-2021), two intensive project weeks and one summer school for advanced students will be conducted. Within these weeks, students shall learn to apply the virtual scanner and record the same object in real in order to compare virtual and real-world procedures and data. In the first instance, real cultural heritage monuments will be recorded onsite and processed since they offer a high geometric complexity often under challenging exterior conditions. As a result, the participants shall conclude improvements for the software environment and contribute to updated e-learning material as well.

Our developed virtual 3D scanner tool, along with the developed e-learning material with interactive tools and practical experiences, allows overcoming previously mentioned limitations like lacking financing and knowledge. Opportunity to acquire the skills to work with the latest tools will lead to the widespread implementation of technology in the production process.

The targets users of the scanner could be:

- Typical Users, such as students, who wants to use VirScan3D to train skills in field phase of terrestrial laser scanning.

- Advanced/Professional users, such as engineers or researches, who want to use VirScan3D for planning of fieldwork in laser scanning or generating artificial point clouds for simulation purposes.

\subsection{State of the art}

\subsubsection{Educational simulation in virtual environment}

During the last 30 years, the virtual environment has become a part of daily real life. Starting with the discovery of virtual particles in quantum physics (Mandl, Shaw, 1984) and continued by the development of such concepts as virtual object/memory/space in computer engineering (Elke, 2004), the term 'virtual reality' became a reflection of the rapid computerization of humanity.

The technology of virtual reality allows interacting with the computer generated world through special graphics and devices. The application of virtual reality is widely spread both, at the domestic level in the form of various video games and in science, solving problems of fundamental disciplines and highly specialized areas.

In the educational sphere, the term of "educational simulation" has been formed, which refers to different environments representing the behaviour of any real objects, systems, phenomena for study and investigation (Aldrich, 2005, Shannon, Johannes, 1976). The ability to reflect and interact with a simulated object ensures the highly effective educational process an adequate framework (Fallon, 2019; Widiyatmoko, 2018).

Thus, in aviation, the virtual cockpits are simulated for the training of future pilots as well as for providing of actual flight information (EASA, 2018; EHEST, 2015). Similar virtual simulators are used for driving training by other vehicles (e.g. the Daimler-Benz-Simulator in Drosdol et al., 1985, Jimenez, 2018), in medicine - for training and carrying out of complex surgical operations (Robertson et al., 2017, Badash et al., 2016) and almost in all branches of architecture and industrial construction (Peruzzini et al., 2018).

In the higher education, the innovative training methods increasingly include video games that have proven a positive impact on learning goals balanced with conventional training methods (Vlachopoulus, Makri, 2017). Especially in geospatial sciences, the simulation in virtual environments is mainly used for the virtual reconstruction of natural scenes like cultural heritage objects or 3D cities and its real time manipulation (Kersten, Edler, 2020; ISPRS, 2020 WG IV/9 Geovisualization, Augmented and Virtual Reality).

Over the years, the application of virtual simulators has fully proven its positive impact for education (aviation - Myers et al., 2018; Socha et al., 2016; Ruiz et al., 2014; medicine - Rehman et al., 2013; Shepherd et al., 2010). Their application is especially necessary in areas, where the training on real objects is related to

high risks to human life and health (plane, etc.), high financial costs for equipment,

- low or impossible accessibility to the real research objects and equipment (e.g. simulation of lost historical objects).

\subsubsection{Tools for virtual scanner realisation and e-Learning}

The digitalisation of teaching has transferred many learning processes to a virtual environment. This is what the creation of virtual classrooms (e.g. $\operatorname{sim} 4 \mathrm{t})$ and the increasingly application of virtual reality gadgets speaks of (Lindner et al., 2019). In the field of higher education, the use of e-Learning tools is a standard training form in many countries. Special learning platforms are applied (e.g. Moodle, Lectora, Panopto), which allow

online learning for educational institutions, management of users, courses, content and activities, interaction with course participants,

documentation of results.

Some platforms make the telepresence of meeting participants possible in VR (Weinmann et al., 2020).

Development of virtual simulators is possible using different commercial and (open source) game engine and 3D modelling 
software (e.g. Unreal Engine, Unity, Blender). Sometimes this is supported using additional gadgets like VR headset and joystick.

In the context of sensor simulation, the BlenSor can be mentioned here as a simulator of various sensors, which allows to simulate the various types of range scanners for researcher in the field of point cloud processing (Gschwandter et al., 2011).

At the moment, the simulation of modern geodesic instruments is rather unusual, but some attempts were made to simulate a classical instrument in virtual environment (Dib et al., 2014; Kuo et al., 2011). Therefore, this DAAD project proves that there is a factual and urgent need to implement and develop such simulators. Next to the sensor simulation, our specific goal is to reconstruct the workflow and interface of popular terrestrial laser scanners (Leica, FARO), which allows the students rapid induction and using of these scanners in the practice.

\section{METHOD}

\subsection{Simulator description}

As mentioned before, VirScan3D is developed for students of geodetic and other higher education departments with limited access to laser scanning equipment. It allows simulating all the processes of the fieldwork phase of terrestrial laser scanning and generates results, which are very close to data generated with real physical scanners on-site. These results can be used for further processing in scanning software at office work phase.

The project is developed using readily available resources and tools and it is designed to run on Windows platform.

The product functionality includes following features:

- selecting the scanner type which will be simulated;

- laser scanning process simulation with visualisation and obtaining point clouds with intensity and noise values according to scanner specifications;

- scan station management, which allows to specify settings for each scan station, create and delete stations;

- target management, which allows placing and removing targets of different types;

- project management, which allows to save the project as a user and to continue work later;

- batch scanning;

- real-time visualisation of generated point clouds;

- exporting point cloud.

The simulator offers two scenarios of work:

1) The user is navigating within the virtual environment and selects target positions as in real life. Then scanner position and scan settings are specified and scanning is started. The scanning process is visualised in real time. When the scanning is finished the user avatar 'goes' to next station.

2) The user navigates within the virtual environment and selects positions for all targets and all scan stations. Subsequently, scan settings are specified for all stations or for each station separately. Finally, the scanning simulation is started for all stations one by one.

Scenario 1 is preferable for new users who have only basic knowledge of laser scanning technology, while the scenario 2 is suited for users who have some experience in laser scanning and want to improve their skills by testing different scanning configurations.

The result of the simulation is a structured $3 \mathrm{D}$ point cloud in ASCII format for import in further software. For each scan station a single point cloud is generated, hence no registration is provided. It is also possible to export target coordinates to use it in registration software for cloud referencing.

\subsection{Realisation}

VirScan3D is developed within Unreal Engine (a well-known gaming engine); therefore, user's computers should satisfy its minimum requirements. However, the speed of scan simulation directly depends on computer performance. Therefore, to obtain the speed of simulation no slower than scanning in real world, the following specifications are recommended:

- OS: Windows 10 64-bit

- Processor: Quad-core Intel or AMD, 2.5 GHz or faster

- RAM: 8 GB RAM

- Video Card/DirectX Version: DirectX 11 or DirectX 12 compatible graphics card.

The minimum hardware requirements of VirScan3D are a 2.0 $\mathrm{GHz} \mathrm{CPU}$ and $4 \mathrm{~GB}$ of RAM. In addition, because VirScan3D uses an OpenGL 3D engine for visualization, a compatible graphics card is required.

Main functions are programmed with Blueprint Visual Scripting system of Unreal Engine. It allows making the system very flexible and modifying software modules without recompiling the whole code. Some specific modules were programmed with $\mathrm{C}++$ language due to Blueprint limitations.

Implementation of advanced features in future versions of software might need the use of additional external software, e.g. Blender.

\section{RESULTS}

\subsection{Presentation of first results}

As a prototype, we used the Leica BLK 360 laser scanner. The prototype of the virtual scanner simulation is presented in Figure 1.

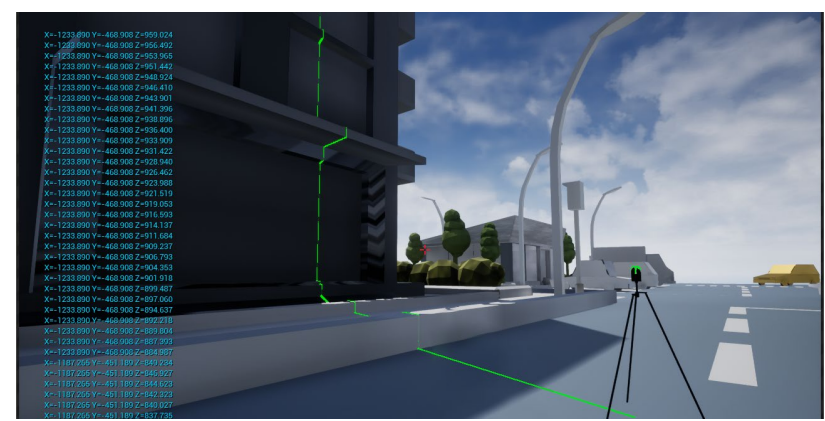

Figure 2: Prototype of the virtual scanner simulation system

In the future, other common laser scanner brands (Faro X330, Leica Scanstation P40) will be simulated to extend the training variety. The process starts with placing of numbered targets and laserscanner positioning (s. Fig. 3). 

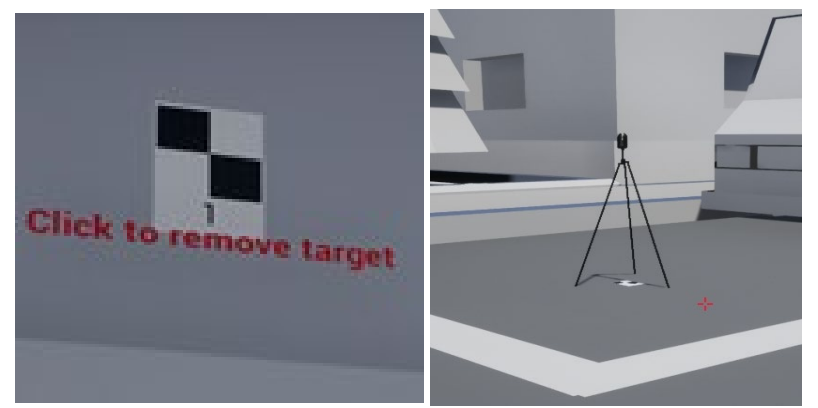

Figure 3: Target placing and laser scanner positioning in VirScan3D software

The figure shows the virtual scanner in action, i.e. a sequentially scanning laser beam is created which hits the surface of the 3D model in order to generate a $3 \mathrm{D}$ coordinates of that point. For the process simulation, we used default device settings for resolution, scan quality ( $1 \mathrm{x}$ beam shooting per point) and time (approx. 15 minutes pro scan station) by clear sky.

The scanning process is simulated graphically so that the user can see the operation in real time (s. Fig. 4). After finishing the simulated scan, the generated $3 \mathrm{D}$ point cloud is exported for further processing by standard software packages via import/export functions.
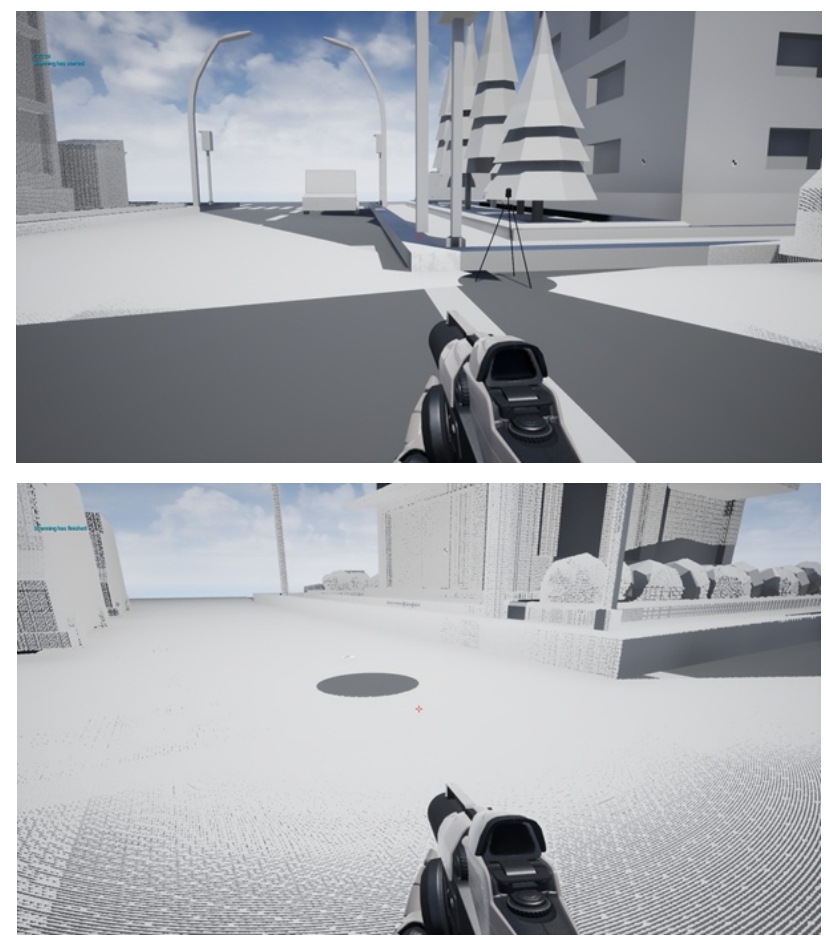

Figure 4: Different scanning periods: top - scanning start, down - end of scanning. The user's gaze and navigation control is simulated by an available device, a gun, but will be replaced by another tool in further development.

After the scanning, a 3D point cloud is generated in Cartesian coordinates $(\mathrm{X}, \mathrm{Y}, \mathrm{Z})$. According to the station settings, angular grid of rays are projected from station point. An intersection of the ray with the first surface gives a discrete point, which XYZ coordinates are stored in text file. The origin of the coordinate system has been set to the station point.
By picking a point on the 3D model a user specifies a scan position. Than the system checks, if the station is correct, and saves it.

There are some functional requirements to be noted:

- A station is incorrect, if it is too close $(20 \mathrm{~cm})$ to other objects or inside of other objects;

- A station is incorrect if it is located on vertical $\left( \pm 30^{\circ}\right)$ surfaces.

During the scanning process XYZ coordinates, distance, horizontal and vertical angles should be stored. Maximum range of surface search is limited to scanner specification. A txt file is to be found in the software directory. Figure 5 represents an example of a single point cloud, generated by the simulator.

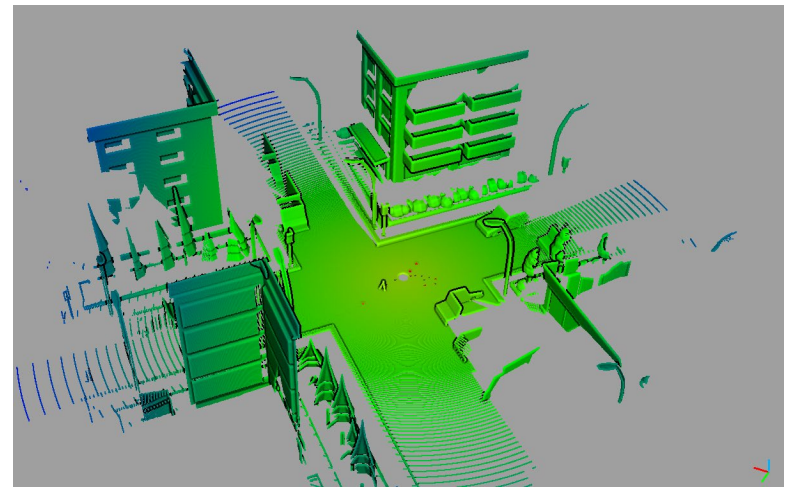

Figure 5: Example of a point cloud generated by the simulator

As mentioned above, the current prototype version consists of only one scanner model and a demonstrational $3 \mathrm{D}$ environment as a reference for simulation. It is expected that release versions of software will have several models of scanners implemented and a possibility to configure custom scanners. A highly detailed precise 3D model of the real environment of the University of Bamberg, based on real laser scanning data, will be used as a reference environment. The functionality to place targets is already implemented in a prototype version, which allows users to register resulting point clouds using a target-totarget approach. Also, the future versions of simulator will be able to simulate the intensity and noise.

The comparison with real data will be distinguished in the future during the 'summer school' application weeks planned in the project period $(2019-2021)$.

\section{CONCLUSION}

\subsection{Evaluation}

The developed software tool allows users to reproduce the sequence of actions at the stage of fieldwork and create realistic data in the absence of a real measuring device. Users can plan their fieldwork in the virtual environment, i.e. specify $3 \mathrm{D}$ scanner type and target positions or change scanner settings. In addition, users can observe the process of virtual scanning and analyse the results, e.g. check the density of the point cloud and surface coverage of the recorded object.

VirScan3D, as new virtual instrument can be used to create 3D point clouds from different stations; in a further step, these clouds have to be registered (geo-referenced) and processed to a 
higher-level information such as 3D models, architectural drawings or maps. The virtual 3D scanner emulates commercially available instruments with realistic user-interfaces and creates $3 \mathrm{D}$ point clouds in a virtual simulation according to individual specifications and settings. Highly detailed and accurate BIM models, which represent real world objects, can be used as training objects. With such a virtual system, teachers as well as students can learn and prepare to operate those systems, generate close-to-reality large data sets and perform the complete chain of data processing.

\section{Summarizing the simulator features, we state that VirScan3D:}

- gives a good 3D laser scanning visualisation for a hands-on process explanation to use a TLS device;

- helps to learn basic laser scanning parameters and interface usage, useful for modelling of an individual workflow;

- allows the generation of scanning results comparable with real data;

- replaces real equipment for practical exercises in case of lacking financing of expensive equipment;

- is a useful tool for distance learning courses.

\subsection{Simulator optimization and further works}

Future work will consist of a more realistic simulation of laser intensity based on

- material-dependent reflection functions resulting as intensity/RGB value and

- estimated beam diameter for the area, where the laser beam hits a surface element (laser spot).

- variety of laserscanning settings specifying such parameters like

- station name

field of view,

resolution,

quality etc.

that should be defined for each scan station.

It is also desired, that users can load their own 3D model in the virtual environment, which is, however, currently not supported by UnReal Engine.

Extensive tests with student groups will demonstrate the performance and usability of the virtual scanner, which will lead to further improvements.

Finally, photogrammetric image simulation can be developed based on the same idea of individual navigation and definition of standpoints/ stations.

\subsection{Possible application of the simulator}

Students and teachers can use the system to learn about the planning, measuring and processing workflows with current equipment without investing in their own (expensive) hardware. Since the virtual data will be generated under consideration of certain noise and outliers similar to a real-world 3D scanner, the possible practical problems with real scanning data become evident and the users must solve them individually. Hence, the concept of engineering-oriented education is addressed to a large extent.

The target group can also be extended to Continued Professional Development and training of employed engineers and architects.

\section{ACKNOWLEDGEMENTS}

The project is funded under the DAAD program for support for the internationalisation of Ukrainian higher education institutions - shaping the digital future together: GermanUkrainian higher education institution collaborations. The financial support is gratefully acknowledged.

Further information is available on the project website: https://iapg.jade-hs.de/projekte/virscan3d

\section{REFERENCES}

ABI research, 2019: Augmented and virtual reality in education. ABI research for visionaries. https://www.abiresearch.com

Aldrich, C., 2005: Learning by Doing. A Comprehensive Guide to Simulations, Computer Games, and Pedagogy in: e-Learning and Other Educational Experiences. San Francisco, Pfeifer.

Allcoat, D., von Mühlenen, A., 2018: Learning in virtual reality: Effects on performance, emotion and engagement. Research in Learning Technology, 26, 2140.

Badash, I., Burtt, K., Solorzano, C., Carey, J. N., 2016: Innovations in surgery simulation: a review of past, current and future techniques. Annals of Translational Medicine, 4(23), 453 -469 .

Blender, 2020: The modelling software. https://www.blender.org

Cabrera, A.G., Martínez, H.P., Vargas, Y.A., 2016: Digital simulation as a tool for transforming the construction industry.

Dib, H., Adamo-Villani N., Garver, S., 2014: An interactive Virtual Environment for Learning Differential Leveling: Development and initial Findings. Advances in engineering education, 4(1), $2119-2126$

Drosdol, J., Kaeding, W., Panik, F., 1985: The Daimler-Benz Driving Simulator. Vehicle System Dynamics., 14 (1-3), 86-90.

EASA, 2018: Certification Specifications for Aeroplane Flight Simulation Training Devices. European Aviation Safety Agency. Retrieved 2018, from: https://www.easa.europa.eu/

EHEST, 2015: Teaching and Testing in Flight simulation training Devices (FSTD), Leaflet HE 10, European Aviation Safety Agency. Retrieved 2015, from: https://www.easa.europa.eu/

Elke, J., 2004: Origin of the Virtual Memory Concept. IEEE Annals of the History of Computing. 26(4), 71-72.

Falloon, G., 2019: Using simulations to teach young students science concepts: An Experiential Learning theoretical analysis. Computers \& Education, 135, 138-159.

Gschwandter, M., Kwitt, R., Uhl, A., Pree, W., 2011: BlenSor: Blender Sensor Simulation Toolbox. Advances in Visual Computing, 2, 199-208.

Hess, M., Garside, D., Nelson, T., Robson, S., Weyrich, T., 2017: Object-based teaching and learning for a critical assessment of digital technologies in arts and cultural heritage. 
International Archives of the Photogrammetry, Remote Sensing and Spatial Information Sciences (ISPRS), 42(2), 349-354.

ISO 9000:2005, 2005: Quality management systems Fundamentals and vocabulary (with future edition ISO 9000:2015).

https://www.iso.org/obp/ui/\#iso:std:iso:9000:ed-3:v1:en

ISPRS, 2020: http://www.commission4.isprs.org/wg9

Jimenez, F., 2018: Intelligent Vehicles: Enabling Technologies and Future Developments. Oxford, Butterworth-Heinemann.

Kersten, T.P., Edler, D., 2020: Special Issue "Methods and Applications of Virtual and Augmented Reality in GeoInformation Sciences". PFG - Journal of Photogrammetry, Remote Sensing and Geoinformation Science, https://doi.org/10.1007/s41064-020-00109-w -.

Kravchenko. J., Luhmann, T., Schultz, R., 2016: Concept and practice of teaching technical university students to modern technologies of 3D data acquisition and processing: A case study of close-range photogrammetry and terrestrial laserscanning. International Archives of the Photogrammetry, Remote Sensing and Spatial Information Sciences, 41(6), 6569.

Kuo, H.-L., Kang, S.-C., Lu, C.-C., Hsieh, S.-H.,Lin, Y.-H., 2011: Using Virtual Instruments to Teach Surveying Courses: Application and Assessment. Computer Applications in Engineering Education, 19, 411-420.

Lectora, 2020: The learning platform. https://www.lectora.de/

Lindner, C., Rienow, A., Jürgens, C., 2019: Augmented Reality applications as digital experiments for education - An example in the Earth-Moon System. In: Acta Astronautica, 161, 66-74.

Mandl, F., Shaw, G., 1984/2002: Quantum Field Theory, John Wiley \& Sons, Chichester UK, revised edition, ISBN 0-47194186-7.

Moodle, 2020: The learning platform. https://moodle.org

Myers, P. L., Starr, W., Mullins, K., 2018: Flight Simulator Fidelity, Training Transfer, and the Role of Instructors in Optimizing Learning Instructors in Optimizing Learning. International Journal of Aviation, Aeronautics, and Aerospace, 5 (1), Art. 6.

Panopto, 2020: The Video Platform for Distance Learning. https://www.panopto.com

Peruzzini, M., Pellicciari, M., Bil, C., Stjepandić, J., Wognum, N., 2018: Transdisciplinary Engineering Methods for Social Innovation of Industry 4.0. Proceedings of the 25th ISPE Inc. International Conference on Transdisciplinary Engineering.

Rehman, S., Raza, S. J, Stegemann, A.P., Zeeck, K., Din, R., Llewellyn, A., Dio, L., Trznadel, M., Seo, Y.W., Chowriappa, A.J., Kesavadas, T., Ahmed, K., Guru, K.A., 2013: Simulationbased robot-assisted surgical training: a health economic evaluation. Int. J. Surg., 11(9), 841-846.
Robertson, J.M., Dias, R.D., Yule, S., Smink, D.S., 2017: Operating Room Team Training with Simulation: A Systematic Review. J. Laparoendosc. Adv. Surg. Tech. A., 27(5), 475-480.

Ruiz, S., Aguado, C., Moreno, R., 2014: Educational Simulation in practice: A teaching experience using a flight simulator. Journal of Technology and Science Education (JOTSE), 4(3), 181-200.

Shannon, R., Johannes, J.D., 1976: Systems simulation: the art and science. IEEE Transactions on Systems, Man and Cybernetics, 9, 723-724.

Shepherd, C. K., McCunnis, M., Brown, L., Hair, M., 2010: Investigating the use of simulation as a teaching strategy. Nursing standard: official newspaper of the Royal College of Nursing, 24(35), 42-8.

Sim4t, 2019: Simulators for Teaching. Supplement of sophisticated teaching laboratory experiments and support material. https://sim4t.com/

Socha, V., Socha, L., Szabo, S., Hana, K., Gazda, J., Kimlickova, M., Vajdova, I., Madoran, A., Hanakova, L., Nemec, V., Puskas, T., Schlenker, J., Rozenberg, R., 2016: Training of Pilots Using Flight Simulator and its Impact on Piloting Precision. Proceedings of 20th International Scientific Conference. Transport Means, 374-379.

Unity, 2020: The game engine. https://unity.com/ru

Unreal, 2020: The game engine. https://www.unrealengine.com

Valentine, R., Padhye, V., Wormald, P. J., 2016: Simulation Training for Vascular Emergencies in Endoscopic Sinus and Skull Base Surgery. Otolaryngol. Clin. North, 49, 877-87.

Vlachopoulus, D., Makri, A., 2017: The effect of games and simulations on higher education: a systematic literature review. International Journal of Educational Technology in Higher Education, 14, Art. 22.

Weinmann, M., Stotko, P., Krumpen, S., Klein, R., 2020: Immersive VR-based Live Telepresence for Remote Collaboration and Teleoperation. In: Th Kersten (ed.). Publikationen der Deutschen Gesellschaft für Photogrammetrie, Fernerkundung und Geoinformation (DGPF) e.V., 29, 391-400.

Widiyatmoko, A., 2018: The Effectiveness of Simulation in Science Learning on Conceptual Understanding: A Literature Review. Journal of International Development and Cooperation, 24, (1, 2), 35-43. 